\title{
Motor imagery training decreases pain on loading in people with moderate Achilles tendinopathy: A preliminary randomised cross-over experiment
}

\author{
J. Debenham ${ }^{1}$, S. Krummenacher ${ }^{1}$, I. Skinner ${ }^{2}$, D. Hince ${ }^{1}$, M. Travers ${ }^{1}$, B. Wand ${ }^{1}$ \\ 1 University of Notre Dame Australia, Fremantle, Australia \\ 2 Graduate School of Health, University of Technology Sydney, Sydney, Australia
}

\section{CORRESPONDING AUTHOR:}

\section{James Debenham}

School of Physiotherapy

University of Notre Dame

19 Mouat Street (PO Box 1225)

Fremantle, Western Australia 6959

Phone: +61894330996

E-mail: james.debenham@nd.edu.au

DOI:

10.32098/mltj.04.2019.08

LEVEL OF EVIDENCE: 2B

\begin{abstract}
SUMMARY
Background. Achilles tendinopathy (AT) is characterised by pain with loading e.g. hopping. Disruption in the working body schema may contribute to pain. We tested this idea by 'training' the working body schema through motor imagery and testing loading pain.

Methods. 6 participants with mid-portion AT entered this preliminary cross-over study. They received two randomised motor imagery training interventions 1) Specific laterality training using images of feet; 2) Sham training using images of the hand. After each condition, they performed 10 submaximal hops and reported their pain intensity (VAS in mm) and Time to Ease (s) afterwards. Participants were blinded to the study hypotheses.

Results. Mean Pain Intensity was significantly lower after specific laterality training compared to sham ( $\beta$ coefficient $=-13.17,95 \%$ CI -21.3 to $-5.0, p=0.005)$. Mean Time to Ease was not significantly different between conditions ( $\beta$ coefficient $=-38.67,95 \% \mathrm{CI}$ - 91.0 to $13.7, \mathrm{p}=0.13)$. The order of testing was not significantly associated with either measure (Pain Intensity: $F(1,9)=0.05, p=0.83$; Time to Ease: $F(1,9)=0.001, p=0.98$ ).

Conclusions. Participants reported less pain with hopping after specific laterality training than after sham training suggesting disruption of the body schema may contribute to pain and disability in Achilles tendinopathy.
\end{abstract}

\section{KEY WORDS}

Achilles tendinopathy; body schema; laterality training; motor imagery

\section{BACKGROUND}

Achilles tendinopathy (AT) is a common overuse condition characterised by localised pain and swelling and associated functional loss (1). While the exact mechanisms by which tendon pain arises remain unclear (2), abnormal loading during activities involving stretch shortening cycles appears to be a primary factor in producing a symptomatic tendon (3), particularly disruption in the way lower limb stiffness is modulated under load (4).
One factor that might contribute to abnormal loading is disruption in the working body schema - the online cortical maps that are used to plan, coordinate and execute movement (5). There is evidence that ongoing musculoskeletal pain may disrupt cortical maps of the painful area (6-8) and influence performance on psychophysical tasks thought to reflect the integrity of the working body schema $(9,10)$.

One way to test if body schema disruption contributes to maladaptive loading in AT is to see if 'training' the work- 
ing body schema decreases pain with loading. Motor imagery tasks such as laterality recognition are thought to reflect the integrity of the working body schema and it has been suggested that laterality training may normalise body schema disruption (11). We sought to exploit this idea in a randomised cross-over experiment and determine if a session of progressive laterality training specific to the lower limb decreased pain with tendon loading. We hypothesised that participants with AT would report less pain with hopping and recover more quickly after laterality training specific to the lower limb than they would after sham laterality training.

\section{MATERIALS AND METHODS}

\section{Study Design}

This randomised, repeated measures, cross-over experiment was conducted at The University of Notre Dame Australia. Institutional ethical approval (REF: $012111 \mathrm{~F}$ ) was obtained and all participants provided informed consent. This project adhered to the Declaration of Helsinski and journal guidelines (12).

\section{PARTICIPANTS}

6 participants diagnosed clinically with mid-portion AT were recruited from the local medical and sporting community between March and August 2013. Inclusion criteria for both groups were age between 18 and 60 years and fluency in English. Specific inclusion criteria for the AT group were a clinical diagnosis of AT based on the following diagnostic criteria; >12-week history of unilateral mid-portion Achilles tendon pain, concordant pain on palpation, pain with or after loading, morning stiffness and a Victorian Institute of Sports Assessment-Achilles (VISA-A) score of $<80 / 100$ (13). As this was a preliminary investigation using a novel testing paradigm which required multiple bouts of hopping, we cautiously limited our inclusion to participants with VISA-A score of $<80 / 100$ in order to minimise unnecessary provocation of pain from the repeated mechanical loading of the Achilles tendon. Participants were excluded if they were pregnant, had received treatment for another lower limb injury or lumbar spine problem in the past 12-months, had a medical condition precluding physical activity, presented with bilateral AT, had undergone previous Achilles tendon surgery, or if they had insertional tendon pain.

\section{Procedure}

Participants were initially screened for eligibility by a research assistant. To confirm eligibility each participant underwent a physical evaluation conducted by an experienced musculoskeletal physiotherapist and completed the VISA-A (14). Eligible participants were then assigned a research number and basic demographic and clinical information were recorded. Prior to training participants practiced a set of 10 hops on the unaffected leg to familiarise them with the task of hopping in time to a metronome.

To control for order effects, a computer generated random sequence was used to assign which condition the participants would be exposed to first. The two conditions were 1) specific laterality training consisting of images of feet or 2) sham laterality training consisting of images of hands.

In the specific condition participants undertook a 20-minute laterality training task using the Recognise ${ }^{\mathrm{TM}}$ App (NOI, Adelaide Australia) run on an iPad. Vanilla photos of feet in various orientations were displayed on the screen and participants were instructed to decide if the displayed image was a left or right foot as quickly and accurately as possible by pressing the 'Left' or 'Right' icon respectively (figure 1). Images were displayed for five seconds each initially, if the participant scored $90 \%$ accuracy $(\geq 36 / 40$ correct responses) the time images were displayed was decreased by one second to a minimum of two seconds. Once participants were $90 \%$ accurate at two seconds, training progressed by utilising more complex images, termed 'context' and 'abstract'

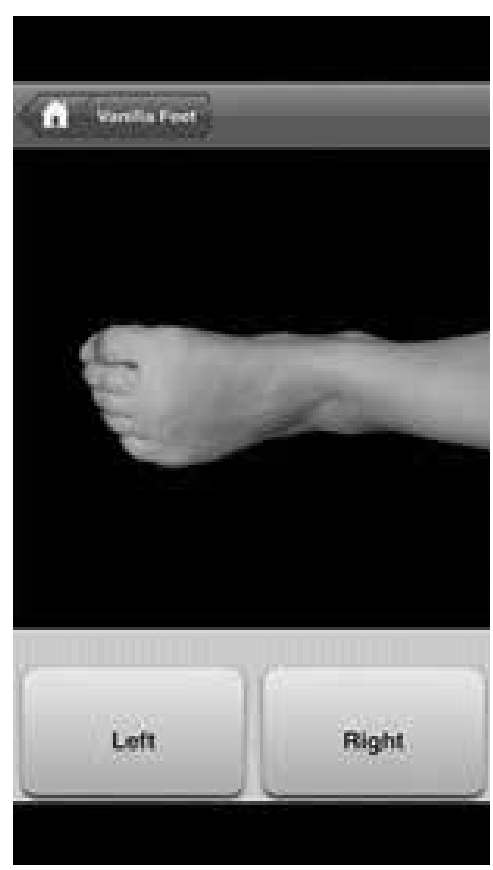

Figure 1. Sample image used for Specific Laterality Training; Reproduced from Recognise ${ }^{\mathrm{TM}}$ App (NOI, Adelaide Australia); note original images were in colour. 
images in the Recognise ${ }^{\mathrm{TM}}$ App. Images were presented in batches of 40 and the rate of progression was individualised depending on performance. Sham laterality training was identical except that photos of hands were used.

Immediately following the completion of the laterality task, the participant was instructed to perform 10 single-leg hops on their affected limb. Participants were positioned with hands on hips standing on a firm surface with no footwear. They were then instructed to hop on the affected leg synchronised to a metronome set at $2 \mathrm{~Hz}(15)$.

After the first condition, participants rested for 20 minutes before undertaking the alternate condition. Randomisation was concealed and participants were blinded to the study hypotheses.

\section{OUTCOME MEASURES}

Pain Intensity was recorded immediately on completion of the hopping task using a visual analogue scale (VAS) anchored with $0=$ "no pain" and $100=$ "pain as bad as you can imagine". Participants were asked to rate their Achilles pain by placing a vertical line at the point on the scale that best corresponded to their pain in that moment. Participant responses were converted to a number by measuring the distance in millimetres from their mark to the left anchor by an independent assessor blinded to condition.

To assess Time to Ease the research assistant started a timer immediately on conclusion of the 10 single leg hops. After completing the post-movement VAS the participant was positioned in sitting and given the timer, and instructed to press stop when their pain had returned to baseline level. The time displayed on the stopwatch was recorded in seconds. If the participant's pain had not eased to baseline within 15 minutes, Time to Ease was recorded as 900 seconds.

\section{DATA ANALYSIS}

A linear mixed model was fitted to Pain Intensity and Time to Ease using the generalized mixed model procedure in SPSS ${ }^{\text {v }} 24$ (IBM Corporation, NY). Both models included task (specific or sham training) and order of testing as fixed terms, and the correlation between responses within the same participant was accounted for by fitting a random effect for participant. $\mathrm{P}<0.05$ was considered significant.

\section{RESULTS}

\section{Participant Characteristics}

All 6 participants completed both the specific and sham training, and there were no missing data. The demographic and clinical characteristics of the participants are shown in table I.

\section{Order of testing}

Order of testing was not significantly associated with either measure (Pain Intensity: $\mathrm{F}(1,9)=0.05, \mathrm{p}=0.83$; Time to Ease: $\mathrm{F}(1,9)=0.001, \mathrm{p}=0.98)$. In addition, removal of order of testing did not alter the parameter estimates for task in either model and thus was not considered further.

\section{Effect of Laterality Training on Pain Intensity}

Figure 2 displays mean Pain Intensity and mean Time to Ease for the two laterality tasks. Mean Pain Intensity score for specific laterality training was $13 \mathrm{~mm}$ lower than that for sham training ( $\beta$ coefficient $=-13.17,95 \%$ CI -21.3 to -5.0 , $\mathrm{p}=0.005)$.

\section{Effect of Laterality Training on Time to Ease}

Mean Time to Ease was on average 38s quicker for specific laterality training compared with sham (figure 2), but this difference was not significant, owing to substantial variability in this measure ( $\beta$ coefficient $=-38.67,95 \% \mathrm{CI}-91.0$ to $13.7, \mathrm{p}=0.13)$. Inspection of the raw data indicated that one participant took 201s for the pain to ease, which was twice the duration of the next highest value.

\section{DISCUSSION}

This study aimed to determine if a session of progressive laterality training specific to the lower limb decreases pain with tendon loading in people with AT. Participants performed either specific laterality training or sham training in random order. Pain on hopping and time for pain to ease were assessed immediately after each training condition. The primary hypotheses of the study were that partici-

Table I. Demographic and clinical characteristics of the 6 participants.

\begin{tabular}{llll}
\hline & n & $\%$ & \\
\hline Gender (Female) & 3 & 50 & \\
\hline Dominance (Right) & 4 & 67 & \\
\hline & Mean & SD & Range \\
\hline Age (years) & 33.67 & 11.06 & $19-53$ \\
\hline Duration of Pain (weeks) & 141.50 & 119.86 & $13-316$ \\
\hline VISA-A score $^{*}$ & 64.67 & 11.76 & $53-80$ \\
\hline
\end{tabular}

VISA A = Victorian Institute of Sports Assessment-Achilles 


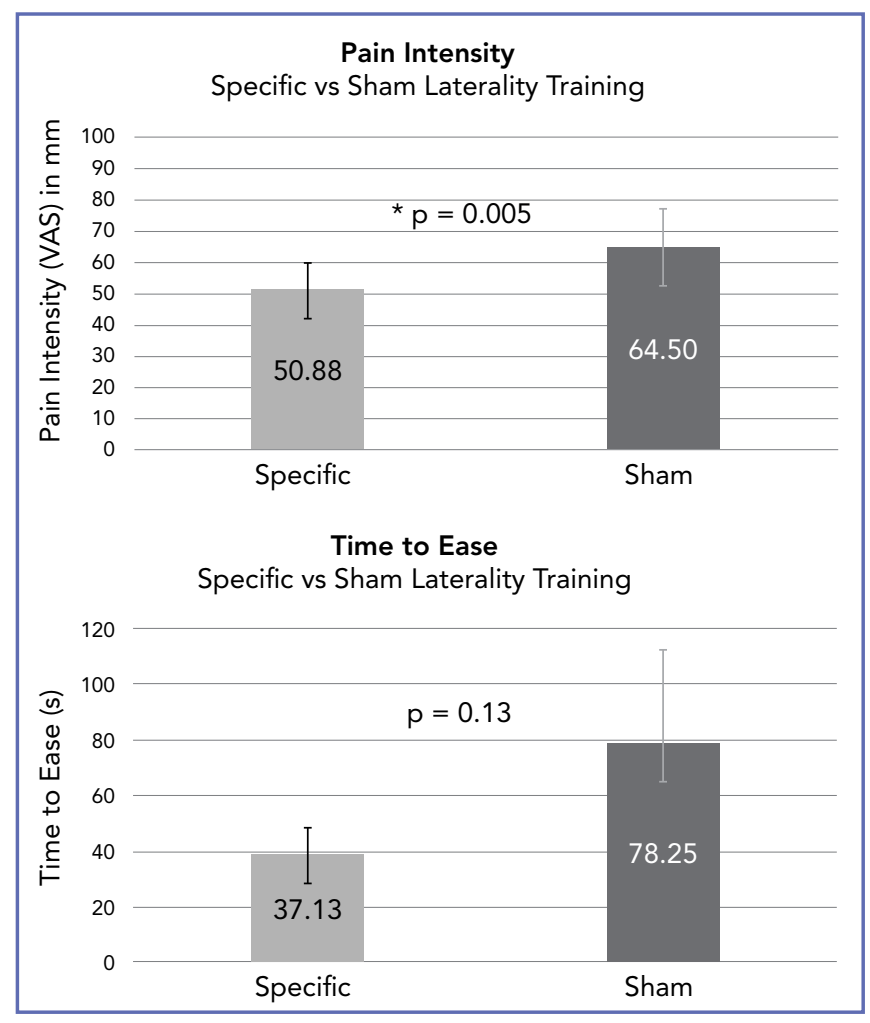

Figure 2. Mean Pain Intensity $(\mathrm{mm})$ and Time to Ease (s) for Specific Laterality Training vs Sham Laterality Training; Error bars represent the standard error of the mean; * indicates a statistically significant difference.

pants would report less pain and recover more quickly after specific laterality training than they would after sham training. In support of our hypotheses participants reported a lower Pain Intensity following specific training. However, no difference in Time to Ease between the two conditions was observed. While the results of small studies must always be interpreted cautiously, the validity of the results is supported by the fact that all subjects reported lower pain scores with specific laterality training.

Statistical analysis revealed that the improvement in Pain Intensity following the experimental condition is not attributed to an order effect. This suggests that the 20 minute wash-out period employed between the experimental and control condition was sufficient. It also indicates that the pain reduction induced by one 20 minute period of laterality training is not sustained beyond this time. This was a desired outcome and highlights the evaluative nature of the protocol employed in this study. As such, we do not recommend use of such a small dose in trials evaluating the efficacy of laterality training as a supplementary management strategy. The application of a protocol that reflects the intensity of previous randomised controlled trials appears necessary (16-18).

This is the only study we are aware of which has investigated the use of motor imagery in AT. However, there is a growing body of evidence supporting similar approaches in a number of chronic musculoskeletal pain conditions $(10,19)$. Evidence of changes in how the affected body part is represented cortically have been a major impetus for the development of these approaches (10), along with psychophysical findings consistent with disruption of the mechanisms that underpin the working body schema (9). Clear evidence of similar impairments in people with AT is lacking, though recent data suggest that in people with unilateral AT, tactile acuity is significantly decreased over the painful tendon (13), an impairment that some suggest may be reflective of disrupted cortical representation of the affected area (20). While it is still unclear if laterality performance is impaired in people with AT, previous research has reported a positive correlation between tactile acuity and laterality performance for healthy controls as well as some, but not all, chronic pain populations (21). The results of the current experiment provides data that laterality performance might be disrupted in chronic AT and as a preliminary study, supports the further exploration of this possibility.

While the extent to which the working body schema is disrupted in AT is as yet unclear, there are plausible reasons as to why this might contribute to symptoms. The most likely explanation is that degradation of the cortical maps used to plan, coordinate and execute movement will alter motor performance and lead to maladaptive tendon loading, thus contributing to the production of nociceptive input from the tendon. It is also possible that disruption of the centrally held representation of the area disrupts the internal calculation of tendon load and contributes to the pain experience via an enhanced threat perception related to incongruence between predicted and actual motor responses $(2,22)$. This preliminary study represents an early indication that strategies that target central nervous system mechanisms may be an effective means to optimise clinical outcomes for lower limb loading injuries, though clearly further studies are required.

\section{CONCLUSIONS}

Participants with AT reported reduced hopping pain after a single session of laterality training using pictures of feet compared to a single session of laterality training using pictures of hands. These preliminary data suggest that alterations in body schema may contribute to the pain and disability associated with AT. This study provides proof of concept to inform further exploration into this area. 


\section{CONFLICT OF INTERESTS}

The Authors declare no conflict of interests.

\section{REFERENCES}

1. Kountouris A, Cook J. Rehabilitation of Achilles and patellar tendinopathies. Best Pract Res Clin Rheumatol 2007;21(2):295316.

2. Rio E, Moseley L, Purdam C, et al. The pain of tendinopathy: physiological or pathophysiological? Sports Med 2014;44(1):923.

3. Munteanu SE, Barton CJ. Lower limb biomechanics during running in individuals with achilles tendinopathy: a systematic review. J Foot Ankle Res 2011;4:15.

4. Debenham JR, Travers MJ, Gibson W, et al. Achilles tendinopathy alters stretch shortening cycle behaviour during a sub-maximal hopping task. J Sci Med Sport 2016;19(1):69-73.

5. Morasso P, Casadio M, Mohan V, et al. Revisiting the Body-Schema Concept in the Context of Whole-Body Postural-Focal Dynamics. Frontiers in Human Neuroscience 2015;9:83.

6. Di Pietro F, McAuley JH, Parkitny L, et al. Primary somatosensory cortex function in complex regional pain syndrome: a systematic review and meta-analysis. J Pain 2013;14(10):1001-18.

7. Di Pietro F, McAuley JH, Parkitny L, et al. Primary motor cortex function in complex regional pain syndrome: a systematic review and meta-analysis. J Pain 2013;14(11):1270-88. doi: 10.1016/j.jpain.2013.07.004.

8. Wand BM, Parkitny L, O'Connell NE, et al. Cortical changes in chronic low back pain: current state of the art and implications for clinical practice. Man Ther 2011;16(1):15-20.

9. Lotze M, Moseley GL. Role of distorted body image in pain. Curr Rheumatol Rep 2007;9(6):488-96.

10. Moseley GL, Flor H. Targeting cortical representations in the treatment of chronic pain: a review. Neurorehabil Neural Repair 2012;26(6):646-52.
11. Priganc VW, Stralka SW. Graded motor imagery. J Hand Ther 2011;24(2):164-8.

12. Padulo J, Oliva F, Frizziero A, et al. Muscle, Ligaments and Tendons Journal. Basic principles and recommendations in clinical and field science research. Muscles, ligaments and tendons journal 2014;3(4):250-52.

13. Debenham JR, Butler P, Mallows A, et al. Disrupted Tactile Acuity in People With Achilles Tendinopathy: A Preliminary Case-Control Investigation. Journal of Orthopaedic \& Sports Physical Therapy 2016;0(0):1-10.

14. Robinson JM, Cook JL, Purdam C, et al. The VISA-A questionnaire: a valid and reliable index of the clinical severity of Achilles tendinopathy. Br J Sports Med 2001;35(5):335-41.

15. Farley CT, Blickhan R, Saito J, et al. Hopping frequency in humans: a test of how springs set stride frequency in bouncing gaits. J Appl Physiol (1985) 1991;71(6):2127-32.

16. Moseley GL. Graded motor imagery is effective for long-standing complex regional pain syndrome: a randomised controlled trial. Pain 2004;108(1-2):192-8.

17. Moseley GL. Is successful rehabilitation of complex regional pain syndrome due to sustained attention to the affected limb? A randomised clinical trial. Pain 2005;114(1-2):54-61.

18. Moseley GL. Graded motor imagery for pathologic pain. A randomized controlled trial 2006; 67(12):2129-34. J Neurology

19. Bowering KJ, O'Connell NE, Tabor A, et al. The effects of graded motor imagery and its components on chronic pain: a systematic review and meta-analysis. J Pain 2013;14(1):3-13.

20. Wallwork SB, Bellan V, Catley MJ, et al. Neural representations and the cortical body matrix: implications for sports medicine and future directions. British Journal of Sports Medicine 2015.

21. Stanton TR, Lin CW, Bray H, et al. Tactile acuity is disrupted in osteoarthritis but is unrelated to disruptions in motor imagery performance. Rheumatology (Oxford) 2013;52(8):1509. 19.

22. Harris AJ. Cortical origin of pathological pain. The Lancet 1999;354(9188):1464-66. 